\title{
TEMPERATURE AND CHIASMA FORMATION IN SCHISTOCERCA GREGARIA
}

\section{AN ANALYSIS OF THE RESPONSE AT A CONSTANT $40^{\circ} \mathrm{C}$.}

\section{S. A. HENDERSON}

Agricultural Research Council Unit of Biometrical Genetics, Department of Genetics, University of Birmingham *

\section{INTRODUCTION}

Received 3.vii.62

Previous attempts to determine the effects of temperature on meiosis, in particular its effects on chiasma formation, have met with variable success. Some workers have claimed a negative correlation between temperature and cross-over or chiasma frequency, others a positive correlation, a bimodal or U-shaped response, a reduction in chiasma frequency at high and low temperatures, or even no effect at all (reviewed by Wilson, I959). The most consistent marked effect induced in plants by high or low temperatures is a reduction in chiasma frequency (Elliot, I955), with asynapsis in extreme cases (Sax, I937; Barber, I94 I, I942; Pao and Li, I948; Dowrick, I957). In Dowrick's case with Tradescantia, the asynapsis was preceded by an initial slight increase in chiasma frequency.

The situation in animals has not been examined very thoroughly. Only two published attempts at determining the effects of temperature on chiasma frequency are available. The first is that of White (I934) who has published chiasma frequency data obtained from individuals of three Acridoid species, Locusta migratoria, Schistocerca gregaria, and Chorthippus (= Stenobothrus) parallelus, maintained at several different temperatures. These data are of limited value, however, for the chiasma frequencies given for each species at every temperature can all be demonstrated to lie within the normal range of variation for these species. In the second case, Elliot (1955) again using Locusta migratoria, was unable to detect any significant changes in chiasma frequency with temperature.

In view of the failure of these attempts to detect such marked effects as have been found in plants, the situation has been investigated more fully, to discover:-(I) whether animal meiosis will also respond to temperature change and, if so (2) whether the response is similar to or different from that obtained in plants and finally (3) to analyse as carefully as possible the nature of the response.

The desert locust (Schistocerca gregaria) was the species chosen for study because of the several advantages known to be possessed by its chromosomes (see John and Henderson, I962). The normal karyotype of this species has already been described in detail by John and Naylor

* Present Address : Department of Genetics, The University, Cambridge. 
( $196 \mathrm{I}$ ). It possesses 23 acrocentric chromosomes-I I pairs of autosomes and an allocyclic sex univalent. The autosomes cover a wide range of size and can usually be individually identified. The present account is mainly concerned with an analysis of the nature of the response throughout the different size groups of the complement. The cytological features and the suggested mechanism by which the observed effects are produced will be dealt with in a separate publication (Henderson, 1962).

\section{MATERIAL AND EXPERIMENTAL TECHNIQUE}

All of the locusts used in this investigation were supplied by courtesy of the Anti-Locust Research Centre, London. They were derived from a relatively inbred culture, started in 1946 , further details of which are provided by John and Naylor (1961).

Young male adults were placed soon after ecdysis in incubators maintained at a constant temperature of $40 \pm 0.5^{\circ} \mathrm{C}$. They were housed in $15 \times 8$ inch cylindrical containers and fed grass daily. No source of light or water was provided.

Two individuals were taken per day of treatment and their testes removed by vivisection. The orange fat body was dissected away with needles in insect saline and the gonad then fixed in $1: 3$ acetic-alcohol. Squash preparations were made, staining with acetic orcein.

Chiasma frequency and position were both scored for every bivalent in 25 cells from each individual sampled.

\section{CHIASMA FREQUENCY RESPONSE WITH TIME}

For the first few days at this high temperature there is no effect on either chiasma frequency or position. This probably explains why

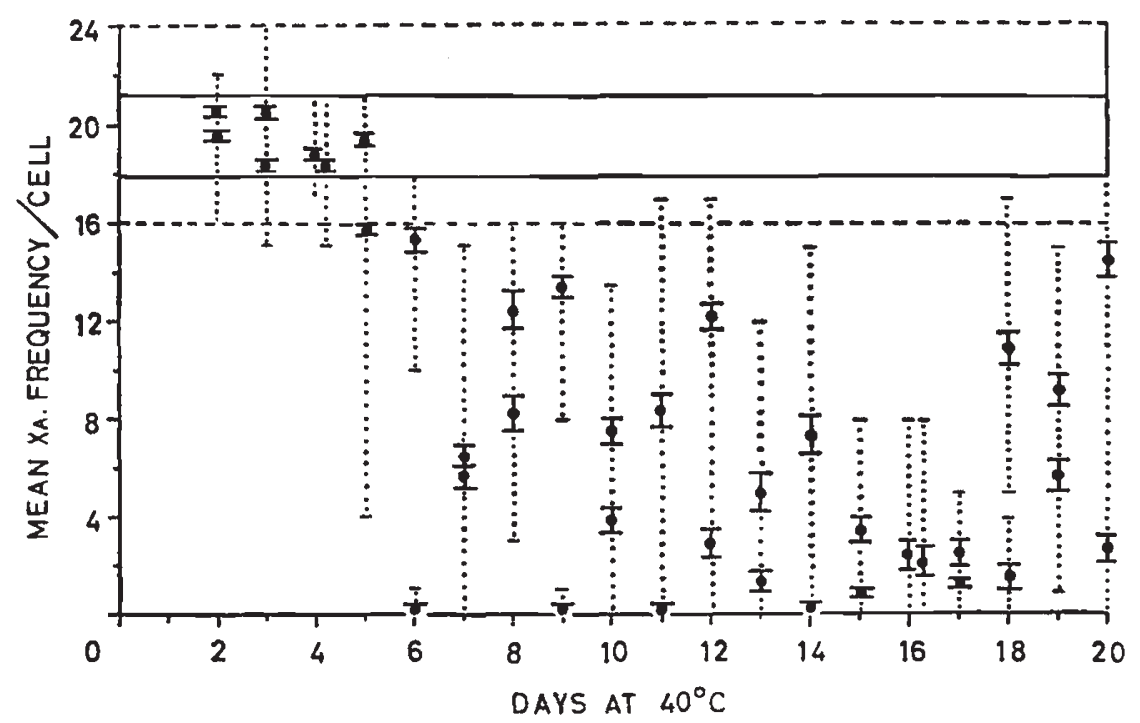

FIG. 10.-Chiasma frequency response with time: mean, standard deviations and ranges are shown for each of the two individuals sampled per day of treatment. Note heterogeneity of reaction. The dotted horizontal lines demarcate the limits of the control range, while the solid horizontal lines show the maximum and minimum control mean chiasma frequency levels. 
White (1934) and Elliot (1955) failed to obtain a response: the durations of their treatments were too short. By the fifth day of treatment a slight reduction in the overall chiasma frequency has been achieved, though this is unaccompanied by any pronounced movements from the normal positions at which chiasmata form or by univalent production. After the fifth day there is a marked reduction in chiasma frequency, which reaches a consistent minimal value at about the seventeenth day of treatment. There is, however, great heterogeneity of response: some "sensitive" individuals achieve total univalence almost immediately after the five-day lag, while others are very much

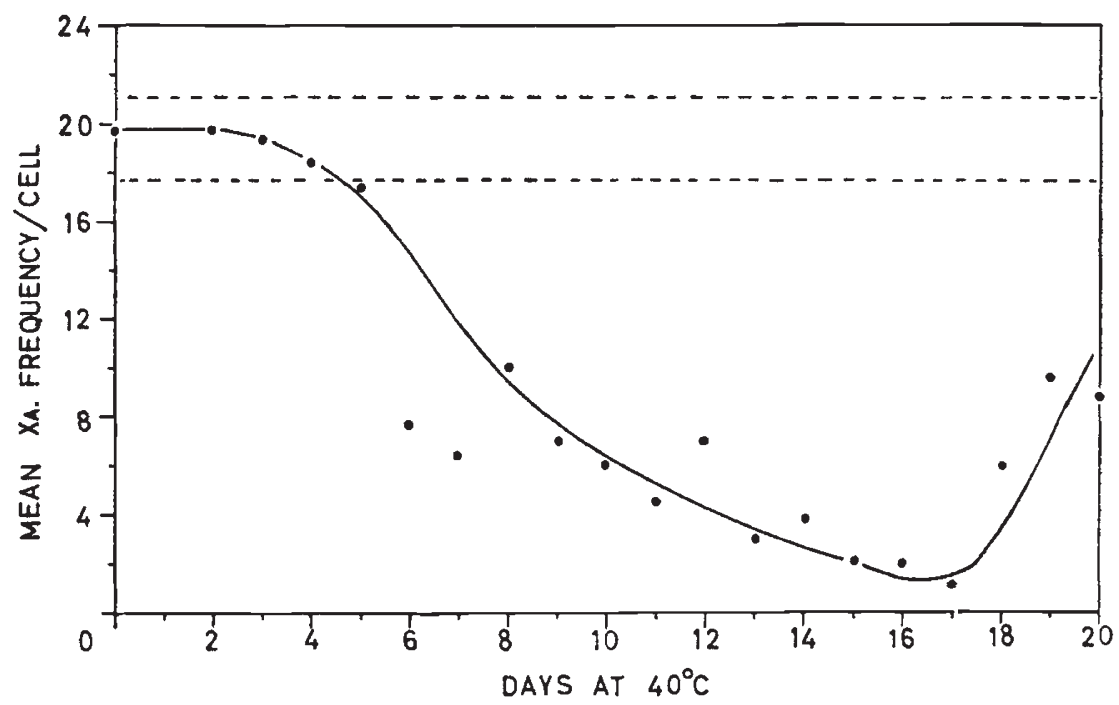

Fro. $1 b$.-Chiasma frequency response with time : overall response clarified on averaging the values obtained from the two individuals taken per day. The levels of the maximum and minimum control means are here shown as dotted horizontal lines.

slower to lose their chiasmata (fig. I $a$ ). Subsequent to the seventeenth day of heat-treatment there is a comparatively rapid partial recovery in most, though not all, individuals. Because of the difficulty in keeping specimens alive at this constant high temperature for very long periods of time, this has not yet been analysed in full. A more detailed appraisal of this recovery will be made at a later date.

This chiasma frequency reduction is accompanied by two features: first, the progressive formation of univalents and secondly, a shift in the position occupied by those chiasmata which do form. There is no initial increase in chiasma frequency comparable to that obtained by Dowrick (1958) in Clone 20 of Tradescantia bracteata.

Many of the effects of individual heterogeneity observed in fig. s $a$ can be removed by taking the means of the two values obtained for each day of treatment. In this manner the time response of the species to the high temperature treatment is clarified (fig. $\mathrm{I} b$ ). 


\section{THE NATURE OF THE RESPONSE}

Because of the great individual heterogeneity (fig. I $a$ ), comparisons made strictly on a time basis necessarily confuse the nature of the response. For the purpose of analysis, therefore, the individuals have been grouped in order of decreasing chiasma frequency. Such
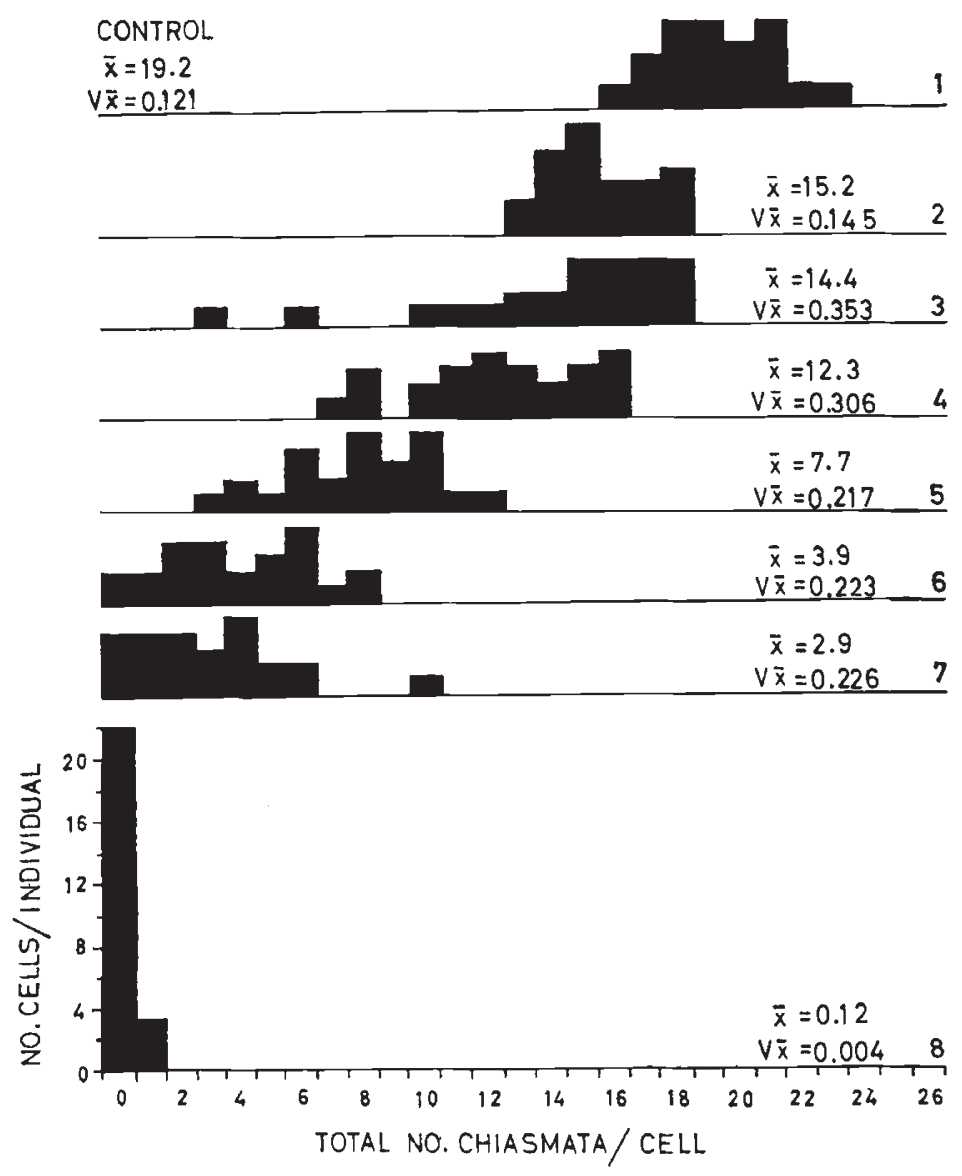

Fici. 2.-Distributions of total numbers of chiasmata per cell for one normal control (1) and seven heat-treated individuals $(2-8)$ arranged in order of decreasing chiasma frequency. Accompanying the shift of the mean $(\tilde{x})$ there is an increase in the variance of the mean $(V \tilde{x})$, in all but the last stages of univalence $(8)$.

grouping illustrates more clearly the nature of the response of the species to decreasing chiasma frequency and increasing univalence. This can be conveniently considered under three general headings.

\section{(i) General chiasma and univalent relationships}

A typical series of histograms demonstrating the effects of increasing univalence on the distribution of total number of chiasmata per cell per individual is provided in fig. 2. The first distribution is from a 
normal control individual. By comparison with this it is seen that accompanying the shift of the mean there is a marked increase in the variance of each distribution. This is maintained until almost total univalence is reached (individual 9) when there is a drastic reduction in the variance with a markedly skew distribution.

With decrease in chiasma frequency there is a progressive increase in the number of univalents present per cell. There are also marked changes in the pattern of the distributions of the number of univalents per cell, which closely fit a binomial expectation. Thus, when the

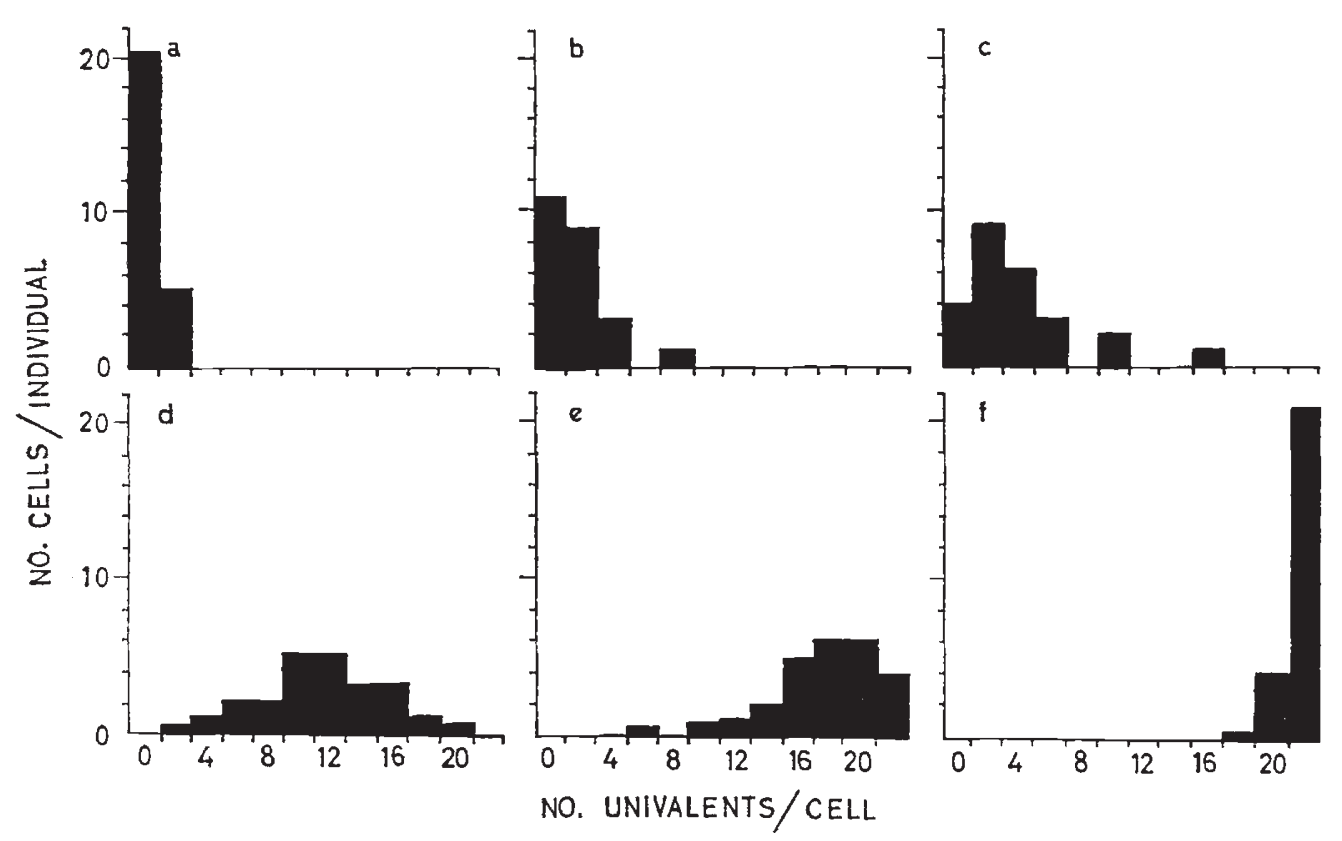

FIG. 3.-Distributions of number of univalents present per cell for six selected heat-treated individuals. These are arranged in order of decreasing chiasma frequency to illustrate the changes in the patterns of the distributions.

number of univalents present is small, the distribution has a strong negative skewness (fig. $3 a$ ). This gradually diminishes, until at $5^{\circ}$ per cent. univalence, a symmetrical distribution obtains (fig. $3^{d}$ ). Increase in the number of univalents present beyond this point produces a positive skewness, which is maximal of course, when virtually total univalence is achieved (fig. $3 f$ ).

If the chiasma frequency per cell is plotted in histogram form against the number of univalents per cell for each of the 25 cells scored per individual, approximately linear relationships are obtained for each individual. However, the slopes differ from one individual to another, depending on the level of univalence. When the number of univalents present is small, the slope is steep; when large the slope is more shallow. A composite histogram, based on 17 individuals, shows the total distribution for all ranges of univalence (fig .4). A 
similar relationship, but with slightly more scatter to the points is obtained if the mean chiasma frequency per individual is plotted against the mean univalent frequency per individual (fig. 5). The

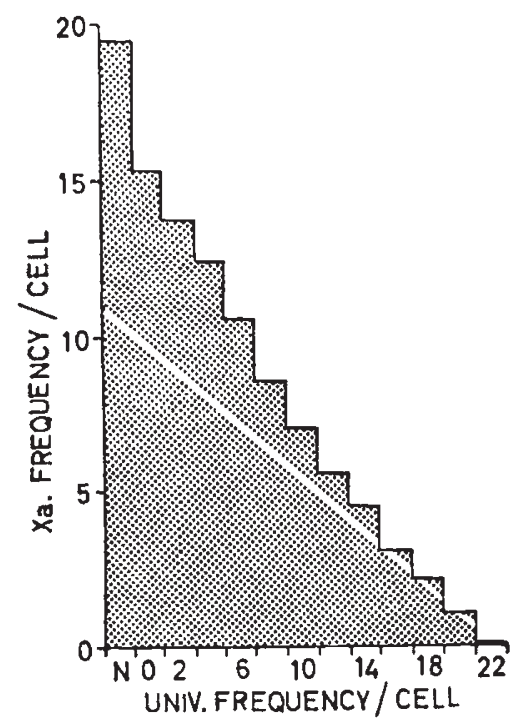

Frg. 4.-Total distribution of univalent frequency per cell plotted in histogram form against chiasma frequency per cell. Based on seventeen heat-treated individuals. A slope of 1 is provided for reference.

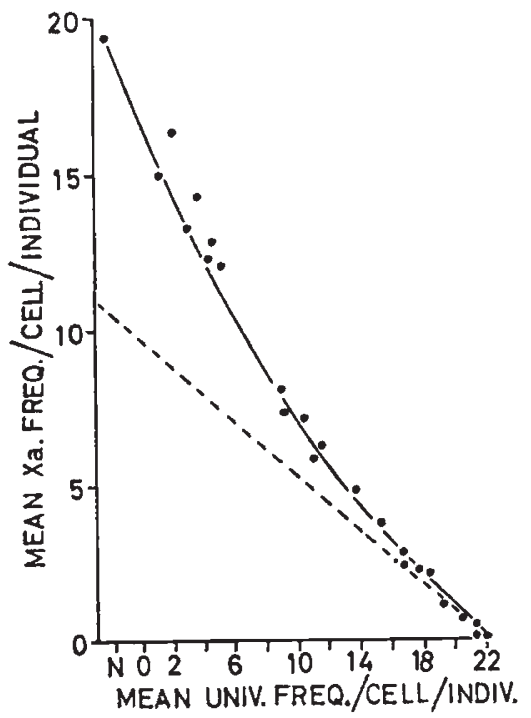

Fig. 5.-Graphical relationship between the mean univalent frequency per individual and mean chiasma frequency per individual. A reference slope of $i$ is again provided.

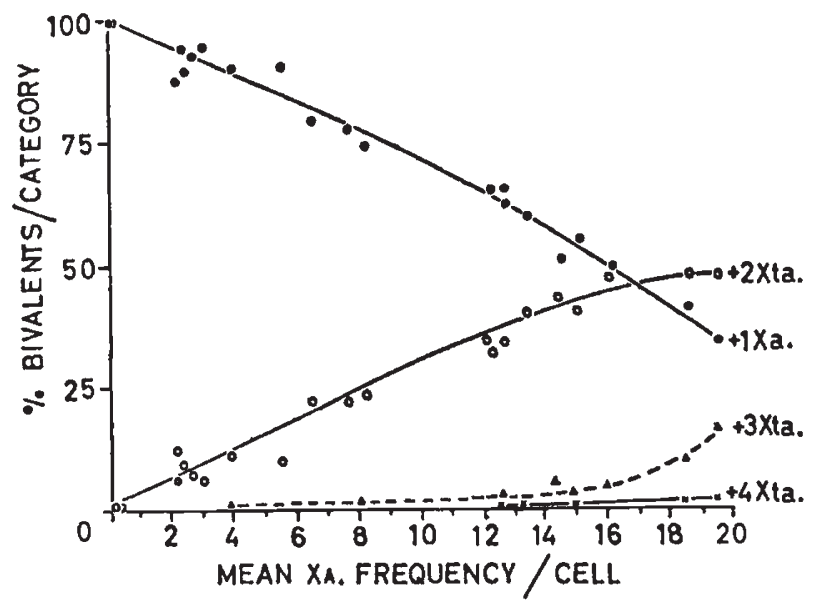

Frg. 6.-Relative frequencies of bivalents possessing 4, 3,2 or I chiasmata, out of those which succeed in forming. These are shown over all levels of chiasma frequency.

relationships are both curvilinear, due to a gradual shift in the number of chiasmata lost per cell in the formation of each additional pair of univalents. Thus, when the number of univalents present is small, there is a comparatively rapid loss of chiasmata. This is in the 
region of two chiasmata per cell per additional pair of univalents. This rate of loss of chiasmata, however, gradually decreases as univalence increases until a slope of $I$ is reached, when one additional pair of univalents corresponds to a loss of only one chiasma per cell.

The manner by which the chiasma frequency is reduced with increasing univalence is summarised graphically in fig. 6. Here the percentages of bivalents possessing $4,3,2$ or I chiasmata out of those which succeed in forming, are plotted against total chiasma frequency per cell. As might be expected, with decreasing chiasma frequency, the number of bivalents forming 4 and 3 chiasmata rapidly drops. Bivalents possessing 2 chiasmata decrease steadily in frequency until total univalence is reached, when their frequency is zero. Bivalents possessing only one chiasma increase proportionately to counterbalance the other reductions.

\section{(ii) Localisation changes}

In control material a single chiasma is most commonly interstitial (I) in position (68 per cent.). Single proximal (P) chiasmata are not usually found, while distally sited (D) single chiasmata occur at a frequency of about $3^{2}$ per cent. When two chiasmata are present, these tend to be approximately proximal-distal in location (P-D) and are never both found at the distal end (D-D).

Accompanying the decrease in chiasma frequency in experimental material there is a shift in the positions in which chiasmata form. This results in their becoming localised towards the chromosome ends, particularly the distal ends of these acrocentric chromosomes. Thus, when only one chiasma forms it is usually distal in location, less commonly interstitial or proximal. When two chiasmata form these are generally proximally-distally localised but both may be sited distally. Two proximally localised chiasmata have never been observed. Three chiasmata per bivalent are only rarely formed in heat-treated cells showing univalence and are P-I-D in location. These marked changes in position are summarised both in tabular form (table 1 ) and graphically (fig. 7). Only the three pairs of long ( $\mathrm{L} I-3)$ and five pairs of medium ( 4 $^{-8}$ ) chromosomes are considered in this analysis because of the difficulty in consistently recording the positions of chiasmata accurately in the three pairs of small (S) chromosomes.

Decrease in chiasma frequency without univalence is shown by individuals $\mathrm{I}$ and 2 , which were subjected to 4 days at $40^{\circ} \mathrm{C}$. These show a marked shift in the positions occupied by single chiasmata. The proportions of those localised distally have risen to $5^{8 \cdot 5}$ per cent. and 73 per cent. respectively, with a corresponding reduction in the percentages of interstitials. Proximally sited single chiasmata are still not found. The percentages of each of the two types of bivalents possessing two chiasmata have not changed much from the control level.

Individuals number $3-22$, which have all been subjected to more than five days of treatment, exhibit varying degrees of univalence and 


\begin{tabular}{|c|c|c|c|c|c|c|c|}
\hline \multirow{14}{*}{ 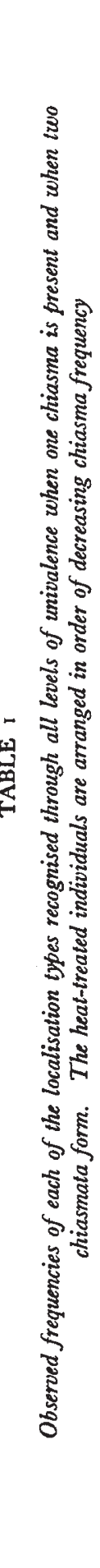 } & \multicolumn{3}{|c|}{ 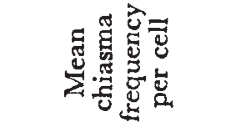 } & $\dot{\omega}$ & $\begin{array}{l}\infty \\
\dot{\infty} \infty \\
\dot{\infty} \infty\end{array}$ & 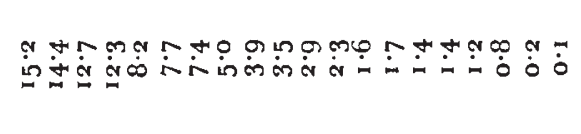 & \\
\hline & \multirow{6}{*}{ 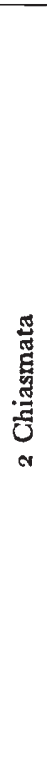 } & \multirow[t]{2}{*}{$\dot{a}$} & 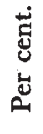 & 1 & $1 \mid$ & $|1| 1|101| 1|1| 1|1| 1 \mid 1$ & $\stackrel{9}{0}$ \\
\hline & & & $\dot{z}$ & 1 & | | & $|1| 1|1-1| 1||||||||||$ & - \\
\hline & & \multirow{2}{*}{ A } & 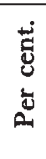 & $\dddot{0}$ & mo & 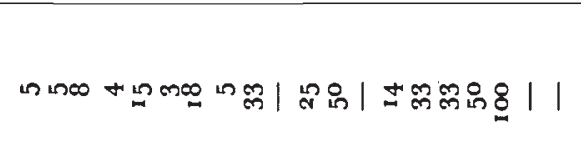 & $\dddot{l}^{\infty}$ \\
\hline & & & $\dot{z}$ & $\dot{0}$ & $+\infty$ & nincomenn-m|m- $|\rightarrow-m-1|$ & ? \\
\hline & & \multirow{2}{*}{$\stackrel{9}{a}$} & $\begin{array}{l}\text { 苞 } \\
\text { 岕 }\end{array}$ & $\hat{\dot{g}}$ & ลิむ & 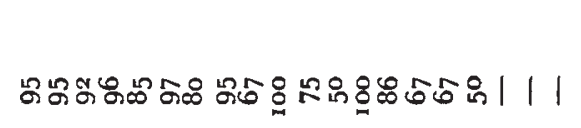 & $\begin{array}{l}\mathscr{m} \\
\text { के }\end{array}$ \\
\hline & & & $\dot{z}$ & ळొ & $\mathscr{\xi}$ & 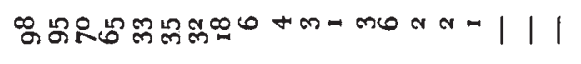 & $\ddot{q}$ \\
\hline & \multirow{6}{*}{ 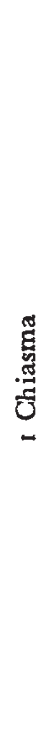 } & \multirow{2}{*}{ 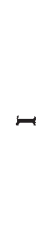 } & $\begin{array}{l}\text { ن. } \\
\text { U⿺ } \\
\text { 峁 }\end{array}$ & $\mathscr{\wp}$ & Fิ & உீ丨刃 & $\stackrel{\infty}{\infty}$ \\
\hline & & & $\dot{z}$ & $g$ & $\therefore=$ & gmagang & 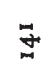 \\
\hline & & \multirow[t]{2}{*}{ a } & 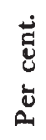 & लै & 品 & 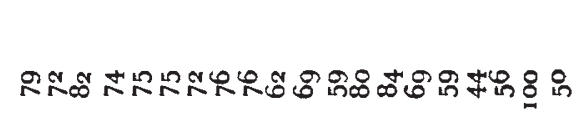 & 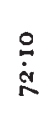 \\
\hline & & & $\dot{z}$ & $a$ & ॠृ & 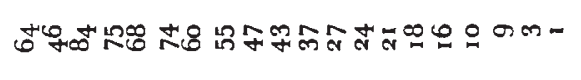 & ले \\
\hline & & \multirow{2}{*}{$A$} & 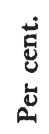 & 1 & | | & 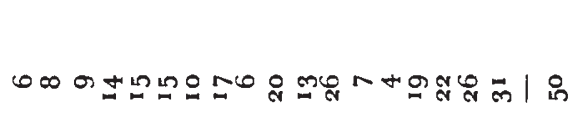 & $\stackrel{\leftrightarrow}{\stackrel{\leftrightarrow}{+}}$ \\
\hline & & & $\dot{z}$ & 1 & 11 & 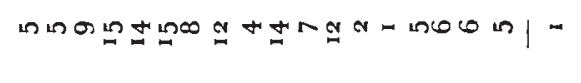 & $\mathscr{F}$ \\
\hline & & \multicolumn{2}{|l|}{ 苞 } & 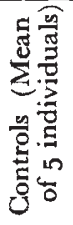 & $\begin{array}{l}\cdot \\
\dot{x} \\
\dot{x} \\
\dot{4}\end{array}$ & 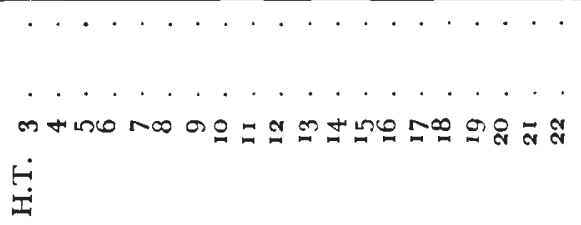 & . \\
\hline
\end{tabular}


are arranged in order of decreasing chiasma frequency. It will be seen that there are some correlations between decreasing chiasma frequency and the relative proportions of each of the different localisation types observed. The percentages of those bivalents exhibiting each of the localisation patterns, out of the total number of bivalents of that chiasma frequency formed, are plotted against the mean chiasma

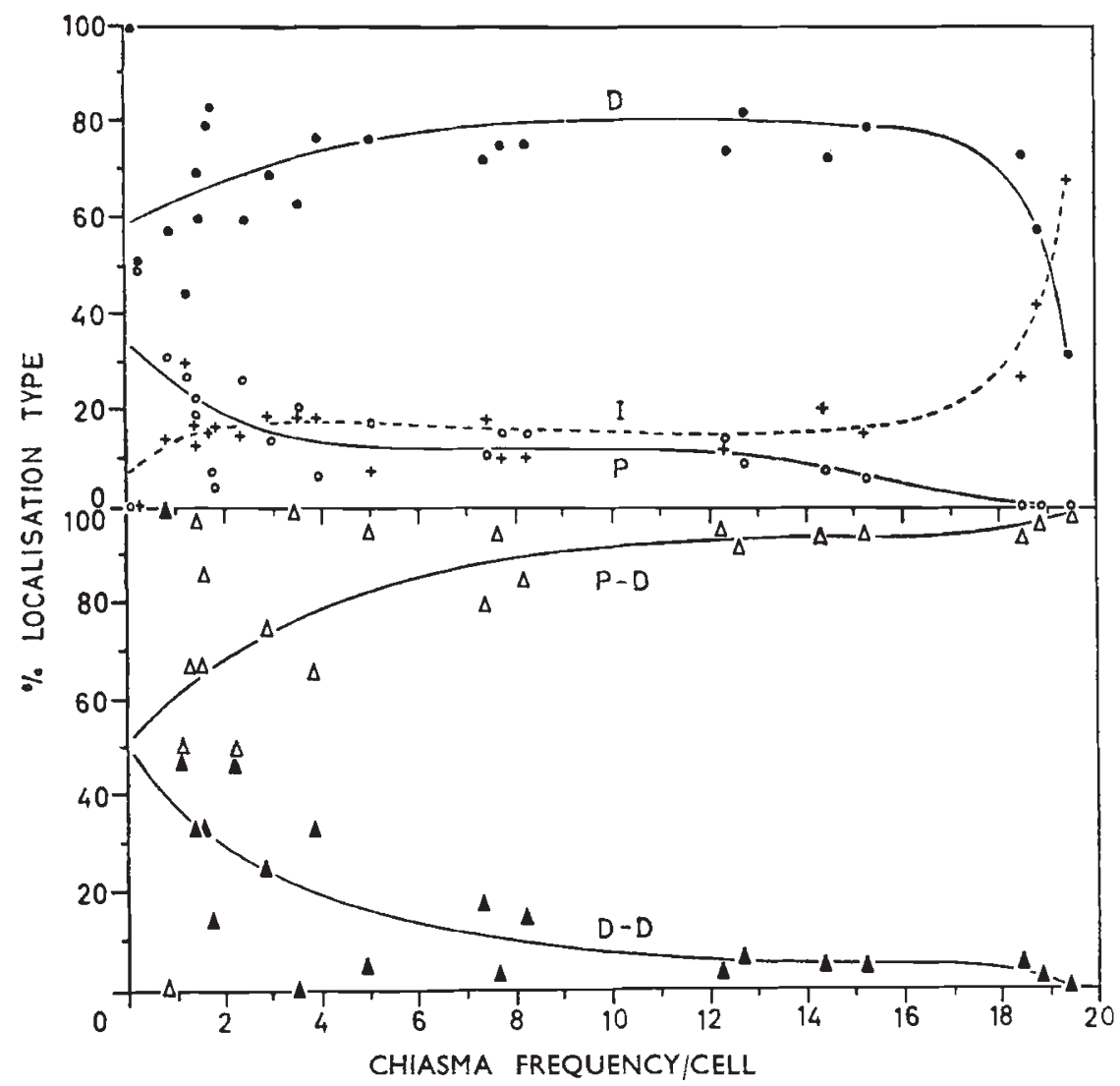

FiG. 7.-Relative frequencies of each of the localisation types recognised through all levels of chiasma frequency in heat-trained individuals, when one chiasma is present (above) and when two chiasmata form (below). $\mathrm{P}=$ proximal ; $\mathrm{I}=$ interstitial ; $\mathrm{D}=$ distal.

frequency per individual in fig. 7 . Single interstitial chiasmata are seen to fall rapidly from the control level of 68 per cent. and remain at a level of $c$. I 6 per cent throughout most ranges of chiasma frequency. On approaching total univalence, however, this class falls below io per cent. Distally localised single chiasmata rise swiftly from the control 32 per cent. to $c .75$ per cent. This is maintained until the chiasma frequency falls below a level of 3-4 per cell, when the percentage obtained is more variable. In some individuals almost ı oo per cent. of the few bivalents forming at this level are of this type; in others the frequency may drop to $c$. 50 per cent. Individual genotypes are probably responsible in part for the great variability observed. But 
one of the main causes is probably the magnitude of the sampling error at very low chiasma frequencies. When only a few bivalents are present in the 25 cells scored, considerable fluctuations in percentages can be produced by a variation of just one or two bivalents. Single proximally localised chiasmata gradually increase in frequency from the control level of near zero to $c$. I 4 per cent. Below a frequency of three chiasmata per cell the percentage of this class increases further still.

Bivalents possessing two distally localised chiasmata, absent from controls, slowly increase in frequency with progressive univalencc. Immediately before total univalence is reached, these increase rapidly in relative frequency, so that many of those few bivalents which succeed in forming two chiasmata are of this type. Pairs of proximally-distally situated chiasmata decrease correspondingly in frequency to counterbalance these changes in the other class.

This preponderance of distal localisation in heat-treated material is in keeping with the localisation affecting some bivalents in the spontaneously univalent individual of this species which has been found (John and Naylor, r $96 \mathrm{r}$ ). This too was to the distal ends of the chromosomes. It is diametrically opposed to the chiasma distribution found in the similar individual of $S$. paranensis studied by John and Henderson ( 1962 ) however; here localisation was proximal.

These fundamental differences between the localisation potentialities of the two sister species are only apparent under conditions of partial univalence. Under normal conditions they are alike in both mean and variance of chiasma frequency, and in the positions occupied by these chiasmata. Two closely related grasshopper species of the genus Keyacris, however, though similar in morphology and in ecological requirements, show localisation differences as a normal feature of meiosis. In $K$. marcida single chiasmata are proximally localised, while in $K$. interpres they are distal in location (White, 1958). Similarly in the sister plant species Allium cepa and A. fistulosum localisation is distal in the former and proximal in the latter. In this case the genetic behaviour has been studied in greater detail. Dominance for distal localisation is revealed by its presence in $F_{1}$ hybrids. The original types resegregate in F2's, but in addition, several new chiasma frequencies and distributions are produced by recombination (Levan, 1936; Maeda, 1937; Emsweller and Jones, 1945). These and similar examples reveal that the region initiating pairing and subsequent chiasma formation can be, and has been, altered in a fundamental manner during speciation. Indeed, there is even evidence from localisation differences which have been found that there may be a comparable major distinction in the regions initiating pairing, between the two sexes of one species. Clearly this is one of the more plastic variables which control chromosome behaviour at meiosis (Darlington, 1940). 
(iii) The differential response throughout the complement

The maximum possible information on the nature of the response throughout the complement was obtained by scoring the chiasma frequencies and positions for each of the bivalents individually. All scorings were made at metaphase I, where, in heat-treated individuals, bivalent recognition is easiest and most reliable (Henderson, I962). In three cases ( $\mathrm{L}_{1}$ and $\mathrm{L}_{2} ; \mathrm{M}_{5}$ and $\mathrm{M} 6 ; \mathrm{S}_{9}-\mathrm{S}_{\mathrm{I}}$ ) individual identification can prove troublesome. Accordingly, in each of these three groups the scores have been pooled and meaned, so that in all, seven size groups are available for comparison $\left(\mathrm{L}_{\mathrm{I} / 2}, \mathrm{~L}_{3}, \mathrm{M}_{4}, \mathrm{M}_{5} / 6, \mathrm{M}_{7}\right.$, M8, S9-I I).

\section{(a) Univalent relationships}

When the chiasma frequency per chromosome pair is plotted against the univalent frequency for that pair in each of the seven size groups,

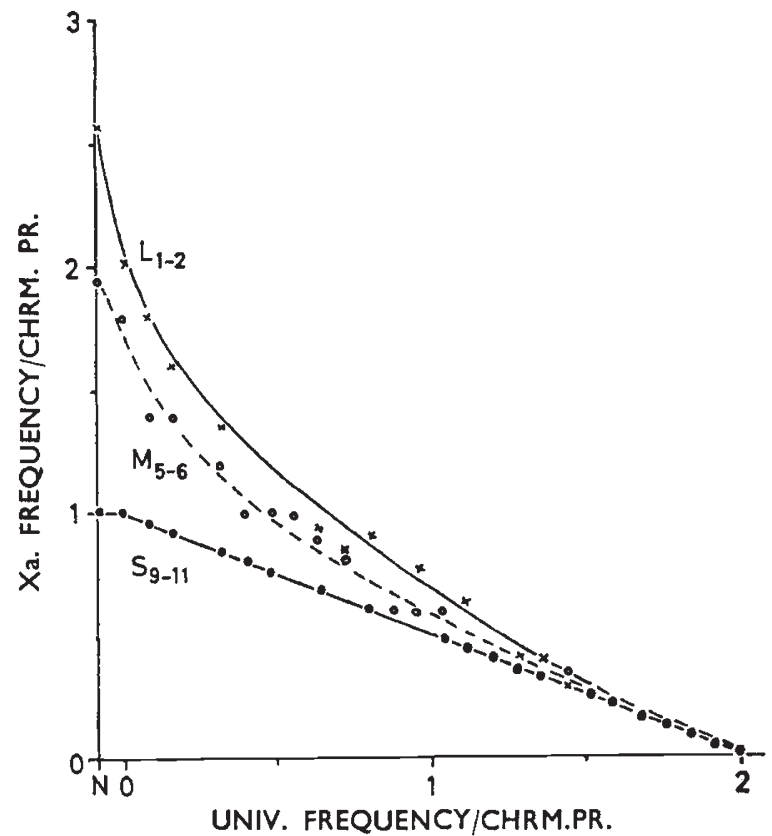

Fig. 8.-Graphical relationships between the chiasma frequency per chromosome pair and the univalent frequency for that pair. Three representative curves are shown, for the long $\left(\mathrm{LI}_{\mathrm{I}} / 2\right)$, medium $\left(\mathrm{M}_{5} / 6\right)$ and short $\left(\mathrm{S}_{9}-\mathrm{I} \mathrm{I}\right)$ size ranges.

curvilinear relationships are obtained for all but the S-group. The composite $\mathrm{LI}_{\mathrm{I}} / 2$ group, which has a control mean chiasma frequency of 2.4 per bivalent, shows a rapid drop in frequency during the initial stages of univalence. Below the level of about one chiasma per chromosome pair, however, the loss is less rapid and more linear. The same kind of relationship holds for all other $\mathrm{L}$ and $\mathrm{M}$ classes, though with decreasing size, of course, the initial chiasma frequency is lower. The three smalls, S9-1 I, have each only one chiasma per bivalent at the outset and for this class the relationship is linear (fig. 8). 
Six selected histograms, showing the distribution of the number of univalents formed throughout each of the seven size groups at various levels of univalence are provided in fig. 9. When the number of univalents present is small, they invariably involve the smaller chromosomes. As the number of univalents per cell rises the long and medium chromosomes are involved to an increasing extent. In most cases the mediums tend to lag slightly behind the longs in the numbers of univalents they form.
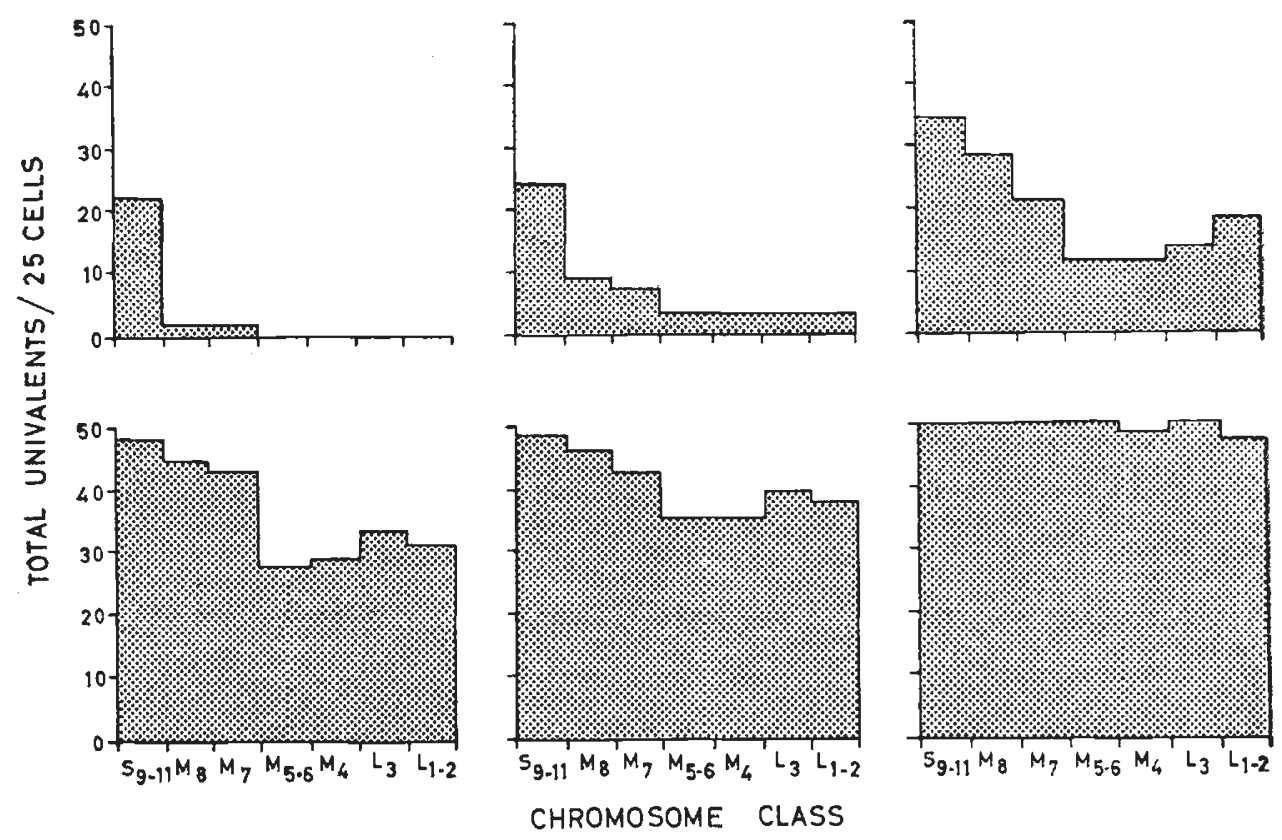

FrG. 9.-Six selected distributions showing the numbers of univalents present in each of the seven size groups of the complement at various levels of univalence. The histograms are arranged in order of decreasing chiasma frequency.

This univalent distribution in heat-treated $S$. gregaria is similar to that obtained by John and Naylor in the anomalous untreated individual of the same species previously cited. Here, too, only the small chromosomes were involved in the slight univalence present. In S. paranensis (John and Henderson, I962) and Locusta migratoria (Rees, 1957), however, the spontaneous univalence was of a different character. Most members of the complement were almost equally involved in the former species, while in the latter example univalents were predominantly formed in the longer size classes. These marked differences are of considerable interest, because in all three locust species similar complements are found-both the chromosome number and the relative size groups present are the same. Furthermore, though the chiasma frequency per cell is significantly lower in Locusta, it is identical in the two Schistocerca spp. 
(b) Chiasma frequency/length relationships

To clarify the distribution of chiasma frequencies throughout the complement the chiasma frequencies for each of the seven recognised size categories have been plotted against their mean mitotic lengths. A representative selection of the chiasma frequency/mitotic length curves so obtained is provided in fig. Ioa. The uppermost group (A)

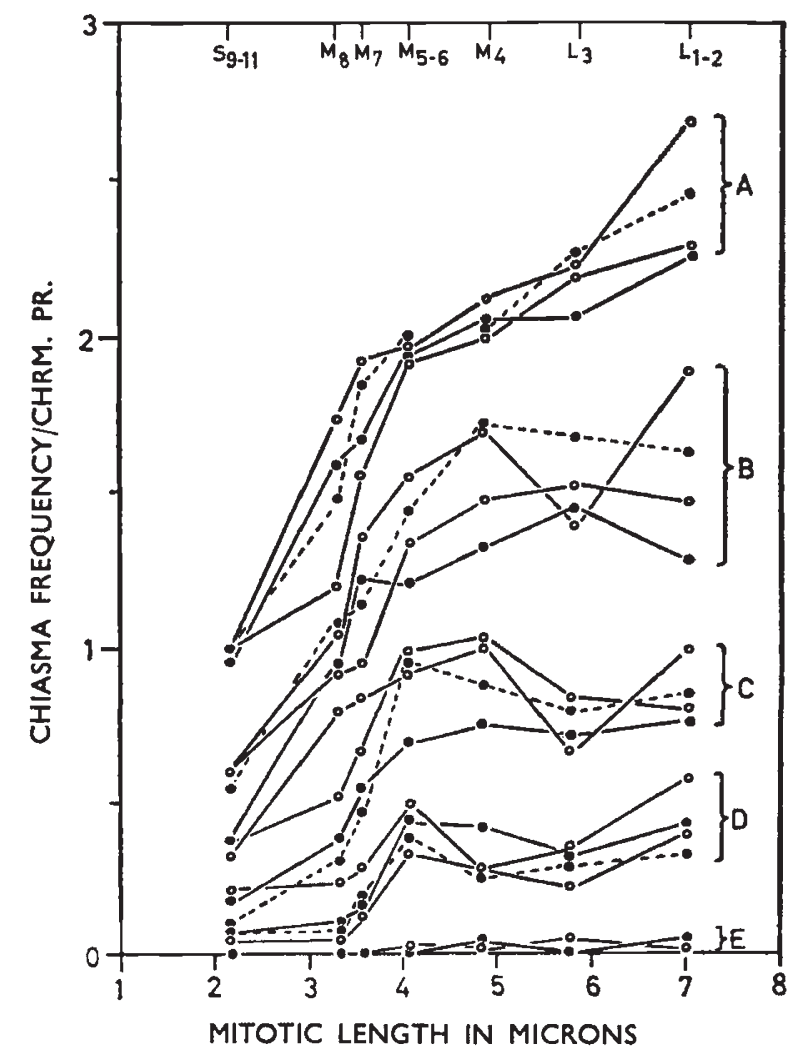

FIG. 10a.-Selected chromosome length/chiasma frequency curves, for the seven size categories recognised, showing the distribution of chiasma frequencies throughout the complement at all levels of univalence. The different symbols used are solely to clarify the distinctions between curves and are of no special significance.

is around the normal, control position. In practice, most of the curves obtained fell more or less around these five groups, but curves occupying all intermediate positions could probably be obtained depending on the genotype of the individual and the duration of the treatment. In this figure, as in the two previous, the pooled bivalent categories are represented by one value only. It must be remembered, however, that these do in fact represent two or three bivalents differing slightly in length and chiasma frequency. Accordingly, in fig. Io $b$ where the mean curves for each of the five groups present in fig. Ioa are shown, the mean values for the two larger pooled classes are given to each of the two chromosomes involved. This indicates 
somewhat more realistically the nature of the changes throughout the complement. This is a satisfactory practice for the $\mathrm{L}_{1 / 2}$ and $\mathrm{M}_{5} / 6$ groupings, for both members possess roughly similar chiasma frequencies. But to do this in the case of the three $\mathrm{S}$ chromosomes would be extremely misleading, for whenever identification is possible the SII always has a lower chiasma frequency than the $\mathrm{S} 9$. For this

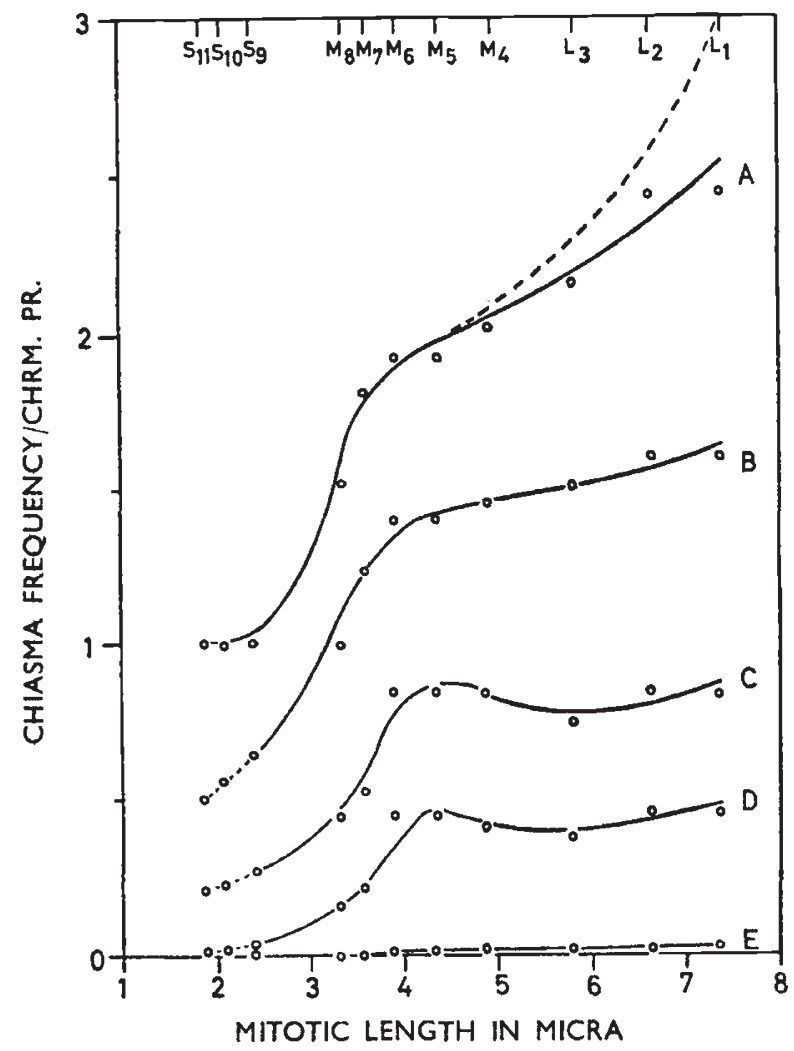

Fia, Iob.-Mean chromosome length/chiasma frequency curves based on twenty-four heat-treated individuals distributed between the five groups shown in fig. 1oa. The mean values for the $\mathrm{L}_{1} / \mathrm{L}_{2}$ and $\mathrm{M}_{5} / \mathrm{M}_{6}$ pooled classes are allocated to the two bivalents involved in each case. The shape of the Sg-1 I section is estimated from unpooled data.

The values for the larger bivalents in curve $A$ are underestimates. The full values for these bivalents, based on the diplotene scoring of 10 control individuals are indicated in dotted outline.

reason the shape of this part of each curve has been estimated and is provided in dotted outline. These positions are based on the individual scorings made for the three chromosomes prior to pooling. Because of the difficulties of identification these figures are not thoroughly reliable on their own, but they do serve as a valid guide for estimation.

Metaphase scorings provide an accurate record of chiasma frequencies in univalent-containing material, for those bivalents which succeed in forming usually possess only one or two chiasmata, rarely three. Curves B-E (fig. $10 b$ ) are, therefore, accurate estimates of the 
chiasma frequency/chromosome length relationships at these levels of univalence. However, in the A group, as in the control material, three chiasmata are often formed in the longer bivalents and occasionally four may be present. When three or four chiasmata are present these are sometimes difficult to recognise at metaphase. Because of this, and possibly slight terminalisation, the values obtained for the longer members of the complement are underestimates. The true chiasma frequency/chromosome length relationship, based on scorings made at diplotene in ten control individuals is shown in dotted outline for comparison.

From these two figs. it may be seen that the initial decreases in chiasma frequency affect the longer half of the complement to a much greater magnitude than the shorter half. However, as the three pairs of $\mathrm{S}$ chromosomes only normally possess one chiasma per bivalent, all reduction, even if slight, results in univalent production in this class. The $\mathrm{L}$ and $\mathrm{M}$ chromosomes are only involved in univalent formation with greater reduction in chiasma frequency. Subsequent reduction continues to affect all classes of chromosomes and the curve, maintaining its flexure, falls to total univalence.

The two points of flexure present enable one, for the convenience of description, to consider the curve as tripartite: the three smalls (S9-I I) lie on the first part, the $\mathrm{M}_{5-8}$ on the second and the $\mathrm{LI}_{\mathrm{I}} \mathrm{M}_{4}$ on the third part.

The flexed shape of these chiasma frequency/chromosome length curves is characteristic for this species and is present even in control curves obtained from untreated material, as can be seen from the dotted outline provided in fig. Io $b$. An analysis of the chiasma distribution at diplotene has recently been made in this species, in which the magnitude and mode of action of the differential and interference distances were investigated (Henderson, I963). In this study it was suggested that the median flexure could result from chiasma formation sometimes beginning at both encis or these chromosomes and proceeding towards the middle. As a result, $\mathrm{ti}$. 2 - $\mathrm{Cr}$. - mosomes would form two chiasmata more commonly than expected and this would raise their chiasma frequencies. Once a consistent mean of two chiasmata per bivalent was exceeded, however, the effect should be lost.

The maintenance of this flexed shape in univalent-containing material, where overall chiasma frequencies are greatly reduced, is rather surprising. The M-chromosomes are shorter than the Lchromosomes. As a result they could be relatively more fully paired than the L's. Under such circumstances it would be possible for chiasma formation to take place from both ends more commonly in the M-than in the L-class. However, while this explanation can be cntertained for those cases where reduction in the overall chiasma frequency is slight or even moderate, it cannot apply to those where a great reduction is found. For in these cases, although only one 
chiasma per bivalent is formed, the $\mathrm{M}_{5}$ and $\mathrm{M} 6$ chromosomes still form them as commonly as the $\mathrm{L}_{1}$ and $\mathrm{L}_{2}$ (fig. rob, D). Clearly, in univalent-containing material, the M-chromosomes have just as high a potential to begin pairing as the L-chromosomes, and can therefore form one chiasma as commonly as the L's. Furthermore, because of their shorter length, pairing can often be completed relatively more fully. Chiasma formation sometimes taking place at both ends can then maintain their frequencies to the values observed through all levels of univalence. The S-chromosomes do not fit in with the behaviour of the remainder of the complement and must therefore be considered to possess somewhat different pairing and chiasma properties.

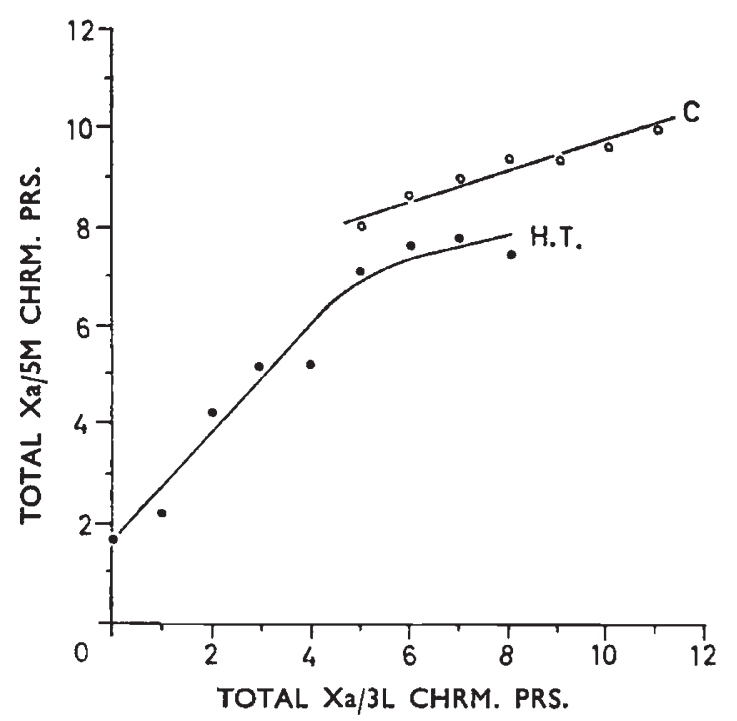

FIG. 11.-Regressions of the mean total numbers of chiasmata per five M-chromosome pairs on the total numbers of chiasmata per three L-chromosome pairs, based on twelve control (C) and twelve heat-treated (H.T.) individuals. Positive correlations are present in both cases.

Finally, the figures were analysed to see if there were any correlations, positive or negative, between the chiasma frequencies of the $\mathrm{L}$ and M size classes ( $c f$. Mather, 1936; Jain and Maherchandani, I96I). The S-chromosomes are, of course, excluded from such an analysis because they do not usually form more than one chiasma. The total number of chiasmata per three L-chromosome pairs ranged between $5^{-}$ II in controls and 0-8 in heat-treated individuals. The total number of chiasmata per five M-chromosome pairs was recorded for each cell and tabulated under the total chiasma frequency of the three L-chromosome pairs of that same cell. This was done for all 25 cells in each of twelve control individuals and twelve heat-treated specimens, covering all levels of univalence. The overall (weighted) mean chiasma totals per five M-chromosome pairs were then taken for each of the L-chromosome chiasma groups. The mean $\mathrm{M}$ chiasma totals obtained in this way were 
plotted against their corresponding L-chromosome chiasma totals (fig. I I). Significant regressions are present in both control and experimental material, which reveal a strong positive correlation between chiasma frequencies in the two size groups; when the Lchromosomes have a high chiasma frequency, so do the M's; when the $\mathbf{L}$ total is low, so is that of the M-chromosomes. Clearly, in this species, those factors determining the chiasma frequency potential affect all bivalents throughout the complement of a cell simultaneously. The stability of the specific chiasma frequency level is here maintained by intercellular control, not by intracellular bivalent compensation.

Rowlands (1958) has suggested that a negative correlation of chiasma frequencies would be obtained if the reproduction of the chromosomes were to be precipitated, or pairing delayed, so that the degree of pairing was reduced. Precipitation of chromosome reproduction with reduction or absence of pairing is exactly what is achieved in heat-treated material. Yet, as can be seen from fig. I , the correlation becomes even more strongly positive in such cells. As Rowlands has pointed out, however, this correlation is not in itself a mechanism for the control of chiasma formation. Rather it is the outcome of such control.

\section{SUMMARY}

I. A constant high temperature of $40^{\circ} \mathrm{C}$. greatly reduces the chiasma frequency at male meiosis in the locust Schistocerca gregaria. In this reduction all levels of univalence may be induced depending on the duration of the treatment and the sensitivity of the individual. For the first five days there is little or no effect on chiasma frequency, but thereafter it falls consistently, reaching a minimal level of almost total univalence by the seventeenth day of treatment. After this time some recovery begins.

2. Coupled with the decrease in chiasma frequency is a change in the position of those chiasmata which do form. This shift is to the chromosome ends, particularly the distal end. Thus, when two chiasmata are present these are P-D or D-D in location, while one chiasma is usually $\mathrm{D}$, less commonly $\mathrm{P}$ or $\mathrm{I}$. At different levels of univalence, however, the relative frequencies of each of these different localisation types varies.

3. Chiasma frequency and position, and univalent frequency were scored for each of the seven size groups recognised within the complement. Detailed analyses were made of the numerous relationships revealed in this way. These involved a consideration of both general chiasma and univalent relationships, and the more specific differential response throughout the different size groups present within the complement.

Acknowledgments.--I am indebted to Professor K. Mather and Dr B. John for their help and encouragement during the course of this work. 


\section{REFERENCES}

BARber, H. N. 1941. Chromosome behaviour in Uvularia. 7. Genet., 42, 223-257. BARBER, H. N. 1942. The experimental control of chromosome pairing in Fritillaria. 7. Genet., 43, 359-374.

DARLINGTON, C. D. 1940. The prime variables of meiosis. Biol. Rev., 15, 307-322. DOWRICK, G. J. 1957. The influence of temperature on meiosis. Heredity, $11,37-49$. ELLIotr, G. G. 1955. The effect of temperature on chiasma frequency. Heredity, 9, 389-398.

EMSWELLER, S. L., AND JONES, H. A. 1945. Further studies on the chiasmata of the Allium cepa $\times$ Allium fistulosum hybrid and its derivatives. Amer. J. Bot., 32, 370-379.

HENDERSON, S. A. 1962. Temperature and chiasma formation in Schistocerca gregaria II. Cytological effects at $40^{\circ} \mathrm{C}$. and the mechanism for heat-induced univalence. Chromosoma (Berl.), 13, 437-463.

henderson, s. A. 1963. Chiasma distribution at diplotene in a locust. Heredity (in press).

JAIN, H. K., AND MAHERGHANDANI, N. 196I. The control of intranuclear distribution of chiasmata in Delphinium. Heredity, 16, 383-392.

JOHN, B., AND hENDERSON, S. A. 1962. Asynapsis and polyploidy in Schistocerca paranensis. Chromosoma (Berl.) 13, $111-147$.

JOHN, B., AND NAYLOR, B. 1961. Anomalous chromosome behaviour in the germ line of Schistocerca gregaria. Heredity, 16, 187-198.

Levan, A. 1936. Die Zytologie von Allium cepa $\times$ fistulosum. Hereditas (Lund.), 2I, 195-214.

MAEDA, T. 1937. Chiasma studies in Allium fistulosum, Allium cepa and their $\mathbf{F}_{\mathbf{1}}, \mathbf{F}_{\mathbf{2}}$ and backcross hybrids. Jap. F. Genet., 13, 146-159.

mather, K. 1936. Competition between bivalents during chiasma formation. Proc. Roy. Soc. B., 120, 208-227.

MAther, K. 1938. Crossing over. Biol. Rev., 13, 252-292.

PAO, W. K., AND LI, H. W. 1948. Desynapsis and other abnormalities induced by high temperature. 7. Genet., $48,297-310$.

REES, H. 1957. Distribution of chiasmata in an 'asynaptic' locust. Nature, $180,589$. RowLANDs, D. C. 1958. The control of chiasma frequency in Vicia faba L. Chromosoma (Berl.), g, $176-184$.

sAX, K. 1937. Effect of variations in temperature on nuclear and cell division in Tradescantia. Amer. J. Bot., 24, 218-225.

WHITE, M. J. D. 1934. The influence of temperature on chiasma frequency. $\mathcal{j}$. Genet., 29, 203-215.

WHITE, M. J. D. 1958. Restrictions on recombination in grasshopper populations and species. C.S.H. Symp. Quant. Biol., 23, 307-317.

WILSON, J. YANNEY. 1959. Chiasma frequency in relation to temperature. Genetica, 29, 290-303. 